\title{
Kallikrein-related peptidase (KLK10) cessation blunts colorectal cancer cell growth and glucose metabolism by regulating the PI3K/Akt/mTOR pathway
}

\author{
H. WEI ${ }^{1}$, C. DONG ${ }^{2}$, Z. SHEN ${ }^{1, *}$ \\ ${ }^{1}$ Comprehensive Administrative Department, The First Hospital of Lanzhou University, Xizhan Branch, Lanzhou University, Lanzhou, Gansu \\ 730050, China; '2Department of General Surgery Ward I, The First Hospital of Lanzhou University, Lanzhou University, Lanzhou, Gansu \\ 730000, China
}

*Correspondence: zhengg_shen@163.com

Received August 14, 2019 / Accepted December 13, 2019

\begin{abstract}
Colorectal cancer (CRC) is a common aggressive carcinoma with a proverbial feature of metabolic reprogramming that is essential for cancer cell growth. Recent research corroborates the controversial function of kallikrein-related peptidase 10 (KLK10) in cancer. However, its role and underlying mechanism in CRC remains elusive. In the present study, high expression of KLK10 was detected in CRC cell lines. Knockdown of KLK10 expression by a specific siRNA inhibited cell proliferation, evoked cell apoptosis, and increased caspase-3 activity in HT29 CRC cells. Furthermore, KLK10 suppression also afforded the suppressive effects on glycolysis in CRC cells as the data showed that targeting KLK10 restrained glucose uptake, lactate production, and glycolysis-related glucose transporter 1 (Glut1) expression. Mechanism analysis corroborated that cessation of KLK10 muted the PI3K/AKT-mTOR signaling. Intriguingly, reactivating the PI3K/AKT-mTOR pathway by its agonist IGF-1 notably reversed the inhibitory effects of KLK10 cessation on CRC cell growth and glucose metabolism. More important, preconditioning with PI3K/AKT inhibitor LY294002 or mTOR inhibitor rapamycin both aggravated KLK10 knockdown-suppressed cancer cell growth and glucose metabolism. These findings suggest that KLK10 silencing may attenuate the progression of CRC by inhibiting cell growth and glycolysis via the PI3K/AKT/mTOR signaling, supporting a potential and promising target for CRC therapy.
\end{abstract}

Key words: colorectal cancer, KLK10, cell growth, glycolysis, PI3K/AKT/mTOR

Colorectal cancer (CRC) ranks as the third most commonly diagnosed malignant carcinoma and the fourth major cause of cancer-related death worldwide. Recently, CRC has become a critical public health threat due to quick increases in incidence and mortality of CRC in regions with a high human development index, such as Asia, Eastern Europe, and South America [1, 2]. By 2030, the occurrence of CRC is estimated to reach 2.2 million new cases and 1.1 million deaths, and the global burden of CRC could increase by $60 \%$ [3]. Despite improvements in clinical prevention and surgical resection, the prognosis of patients with CRC is still poor. To date, the survival rate beyond one-year after CRC diagnosis is less than $50 \%$, and the 5 -year survival is gloomy and less than $10 \%$ [4].

Energy metabolism is often altered and reprogrammed in neoplastic cells including colorectal cancer cells $[5,6]$. Under normal conditions, cells obtain energy from glucose majorly via oxidative phosphorylation; however, tumor cells consume glucose to generate lactate, which is referred to as the Warburg effect (aerobic glycolysis). Reprogramming the metabolic pathway of aerobic glycolysis is consistently accepted as a key hallmark of cancer [5]. Accumulating evidence has corroborated that cancer cells are highly dependent on glycolysis to generate ATP that must be maintained to support cancer cell growth [6,7]. High expression of glycolysis-related genes, such as glucose transporter 1 (GLUT1), have been found in several carcinoma tissues $[8,9]$. Intriguingly, recent research has identified a potential strategy for the treatment of cancer by lowering the metabolic pathway of glucose [10,11].

Kallikrein-related peptidase 10 (KLK10), a member of the KLK family, locates on chromosome 19q13.4 and contains six exons with a length of $5.5 \mathrm{~kb}$. KLK10 was initially identified in 1996 as normal epithelial cell-specific 1 (NES1). Increasing evidence has confirmed the aberrant expression of KLK10 in several cancers, including pancreatic ductal adenocarcinoma, breast cancer, and gastric cancer $[12,13]$. However, abundant study substantiates the contradictive roles of KLK10 in the development of cancers by regulating cell growth, invasion, 
and apoptosis [13, 14]. For research on pancreatic ductal adenocarcinoma, high expression of KLK10 is significantly correlated with poor prognosis and shorter survival, and its knockdown notably suppresses cancer cell invasion and metastatic potential [13]. Conversely, there is low expression of KLK10 in prostate cancer tissues and cells, and its overexpression represses cell proliferation and glucose metabolism [15]. In esophageal cancer, KLK10 elicits a tumor-suppressive role due to its downregulation induces S-phase arrest and promotes cell apoptosis [14]. Recent clinical evidence confirms that KLK10 expression is significantly associated with TNM stage in colorectal cancer [16]. Moreover, patients with high KLK10 levels suffer from shorter disease-free intervals and overall survival rates in colorectal cancer [16, 17]. Unfortunately, the role and mechanism of KLK10 in the development of CRC remain poorly defined.

In the current research, we determined the expression of KLK10 in CRC cell lines. Furthermore, the functions of KLK10 knockdown in CRC cell growth and glucose metabolism were investigated. Additionally, the underlying molecular mechanism also was elucidated.

\section{Materials and methods}

Cell lines and culture. Normal human colorectal epithelial cells (CCD-18Co) and CRC lines including HT29, SW480, and DLD1 were purchased from the American Type Culture Collection (Manassas, VA, USA). Human colorectal cancer cell line HCT116 was purchased from the Chinese Academy of Science Type Culture Collection (Shanghai, China). For culturing, CCD-18Co cells were maintained in Eagle's minimum essential medium (EMEM), and CRC cells were cultured in RPMI-1640 medium. For incubation, 10\% $\mathrm{v} / \mathrm{v}$ fetal bovine serum and antibiotics including $100 \mathrm{U} / \mathrm{ml}$ penicillin and $100 \mu \mathrm{g} / \mathrm{ml}$ streptomycin were supplemented. Cells were grown at $37^{\circ} \mathrm{C}$ in a humidified atmosphere with $5 \% \mathrm{CO}_{2}$ and $95 \%$ air (normoxia).

Knockdown of KLK10 by specific siRNA transfection. To knock down the expression in CRC cells, the specific sequences targeting KLK10 (\#AM16708) and the scramble siRNA (\#AM4611) were synthesized from Thermo Fisher Scientific (Waltham, MA, USA). HT29 cells were then transfected with $50 \mathrm{nM}$ of siRNA using Lipofectamine 2000 (Invitrogen, Carlsbad, CA, USA). Cells transfected with scramble siRNA were defined as the negative control group. After that, the efficacy of si-KLK10 was evaluated by qRT-PCR and western blotting at $48 \mathrm{~h}$ post transfection.

RNA preparation and quantitative RT-PCR (qRT-PCR). Total RNAs from CCD-18Co and CRC cells were extracted using Trizol ${ }^{\oplus}$ reagent (Invitrogen). The first-strand cDNA then was synthesized from the prepared total RNA using the SuperScript II First Strand Synthesis System (Invitrogen) according to the directions of the manufacturer. Subsequently, the real time-PCR was conducted on an ABI PRISM 7000 sequence detection system (Applied Biosystems, Foster City, CA, USA) to quantify the transcriptional levels of KLK10 and Glut1 using the SYBR ${ }^{\circledast}$ Premix Ex Taq ${ }^{\mathrm{Tm}}$ II (Takara, Otsu, Japan). For reaction, specific primers were designed and synthesized by Shanghai Sheng Gong Biological Company (Shanghai, China). The primers used for amplification were as follows: KLK10 (sense, 5'-CCTCCACACCTCTAAACATCTC-3'; anti-sense, 5'-CTTCCTGGCTTCTCTGGAATAA-3') and glut1 (sense, 5'-AGCTACCCTGGATGTCCTAT-3'; antisense, 5'-GAAGAGTTCAGCCACGATGA-3'). $\beta$-actin was introduced as internal quality control to normalize gene expression. To quantify the changes in expression, the method of $2^{-\Delta \Delta C t}$ was applied.

Western blotting. Cells transfected with si-KLK10 were treated with insulin-like growth factor-1 (IGF-1) (PI3K/Akt agonist, $5 \mu \mathrm{M})$, PI3K/AKT inhibitor LY294002 or mTOR inhibitor rapamycin $(10 \mu \mathrm{M}$, Sigma; St Louis, MO, USA). Then, cells were collected and lysed with lysis buffer. The BCA Protein Assay Kit (Beyotime, Shanghai, China) was applied to detect the extracted protein concentration. For immunoblotting, equivalent quantities of protein were loaded onto $12 \%$ SDS-PAGE gels to separate proteins, which were subsequently electroblotted onto a PVDF membrane (Millipore, Billerica, MA, USA). Following incubation with 5\% non-fat milk to interdict the non-specific binding, the membrane was treated with primary antibodies against KLK10 (sc-100551, 1:1000, Santa Cruz, CA, USA), PCNA (sc-25280, 1:500, Santa Cruz), p-AKT (ab38449, 1:4000, Abcam, Cambridge, UK, USA), AKT (ab8805, 1:2000, Abcam), p-mTOR (ab109268, 1:5000, Abcam), and mTOR (ab2732, 1:2000, Abcam). Subsequently, the membrane was incubated with horseradish peroxidase-conjugated secondary antibodies (1:3000, ab6728, ab6721, Abcam) for $2 \mathrm{~h}$. Then, the binding signals were visualized using an ECL kit (Pierce Biotech., Rockford, IL, USA). The intensities of the immunostained protein were analyzed using a Gel Doc ${ }^{\mathrm{TM}} \mathrm{XR}$ imaging system (Bio-Rad, Hercules, CA, USA) and quantified by NIH ImageJ software. For normalization, $\beta$-actin was introduced as an endogenous control.

Evaluation of cell viability. For cell viability assessment, a 3-(4,5-dimethylthiazol-2-yl)-2,5-diphenyltetrazolium bromide (MTT) assay was performed. Cells were seeded into 96-well plates and then treated with si-KLK10, IGF-1, or LY294002. Subsequently, cells were incubated with $20 \mu \mathrm{l}$ of MTT $(5 \mathrm{mg} / \mathrm{ml}$, Sigma). Approximately $4 \mathrm{~h}$ later, the produced formazan crystals were dissolved by supplementing $100 \mu \mathrm{l}$ of DMSO for further incubation for $10 \mathrm{~min}$. Ultimately, absorbance at $570 \mathrm{~nm}$ was captured by a spectrophotometer to assess cell viability.

Assessment of cell apoptosis by flow cytometry. After treatment with the indicated conditions, cell apoptosis was evaluated by the commercial Annexin V-FITC Apoptosis Detection Kit (Beyotime) according to the protocol provided by the manufacturers. Briefly, cells under various treatments were trypsinized and rinsed with PBS buffer. Then, cells were suspended with $195 \mu \mathrm{l}$ of binding buffer. After that, cells 
were incubated with Annexin V-FITC $(5 \mu \mathrm{l})$ and PI $(10 \mu \mathrm{l})$ avoiding light. Fifteen minutes later, samples were analyzed using a FACScan flow cytometer (Becton Dickinson, Sunnyvale, CA, USA) to evaluate cell apoptosis.

Detection of caspase- 3 activity. The activity of caspase- 3 in various groups was analyzed as per the standard protocols of caspase-3 Fluorescent Assay Kits (Clontech; Mountain View, CA, USA). Cells were treated with si-KLK10, IGF-1, or LY294002 and then were harvested. After lysing with lysis buffer, cells were centrifuged and the supernatants were collected. After that, $5 \mu$ of the specific substrate for caspase-3, DEVD-AFC, was supplemented into supernatant for $1 \mathrm{~h}$ incubation. The specimens were then measured by the FL600 fluorescent reader (Bio-Tek Instruments, Winooski, VT, USA) to capture the fluorescence intensities.

Glucose uptake assay. Cells were transfected with si-KLK10 and stimulated with IGF-1 or LY294002. Then, cells were seeded into 96-well plates. Glucose uptake was determined using the commercial Glucose Uptake Colorimetric Assay Kit (Sigma) according to the manufacturer's instructions. Briefly, cells were washed with PBS for 3 times, and glucose-starvation was performed by adding $100 \mu$ of Krebs-Ringer-HEPES (KRH) buffer for $40 \mathrm{~min}$. Then, $10 \mu \mathrm{l}$ of $10 \mathrm{nM} 2$-deoxyglucose (2-DG) was supplemented into each well. Cells were dissolved with extraction buffer, the Reaction Mix A and B were subsequently added. Ultimately, all specimens were analyzed by microplate reader to measure absorbance at $412 \mathrm{~nm}$ to evaluate the glucose uptake level.

Evaluation of lactate production. The content of lactate generation was measured using the Lactate Assay Kit (Sigma) in line with the recommendation of the manufacturer. In brief, cells under various treatments were harvested and homogenized in the Lactate Assay Buffer. Then, cells were centrifuged at for $10 \mathrm{~min}$ and deproteinized with a $10 \mathrm{kDa}$ MWCO spin filter to remove lactate dehydrogenase. Afterward, the extracted soluble fraction was mixed with $50 \mu$ of Master Reaction Mix containing $46 \mu \mathrm{l}$ of lactate assay buffer, $2 \mu \mathrm{l}$ of lactate enzyme mix, and $2 \mu \mathrm{l}$ of lactate probe. After reaction for $30 \mathrm{~min}$, the absorbance at $570 \mathrm{~nm}$ was measured to assess lactate production.

Measurement of intracellular ATP contents. Cells from various groups were lysed with lysis buffer. Then, $50 \mu \mathrm{l}$ of supernatant from each sample was collected and treated with an equal volume of CellTiter-Glo Reagent. After incubation for $10 \mathrm{~min}$ at room temperature, the intracellular ATP levels were determined according to the instructions of the Cell Titer-Glo Reagent (Promega, Madison, WI, USA).

Statistical analysis. All experiments were conducted at least 3 times, and data are presented as the mean \pm standard deviation (SD). Statistical analysis was conducted using SPSS 19.0. Difference comparisons among three or more groups were evaluated using one-way ANOVA followed by the Student-Newman-Keuls (SNK) post hoc test. Statistical significances were inferred at $\mathrm{p}<0.05$.

\section{Results}

High expression of KLK10 is validated in colorectal cell lines. To investigate the function of KLK10 in colorectal cancer, we detected its levels in colorectal carcinoma cells. qRT-PCR corroborated the high transcripts of KLK10 mRNA in CRC cell lines (HT29, HCT116, SW480, and DLD1) relative to normal human colorectal epithelial cell CCD-18Co (Figure 1A). Additionally, compared with CCD-18Co, the protein expression of KLK10 was higher in colorectal cancer cells (Figure 1B).

The knockdown of KLK10 suppresses cell proliferation and evokes cell apoptosis in HT29 cells. To dissect the function of KLK10 in the CRC progression, we explored its cessation in CRC cell growth. As shown in Figure 2A, the siRNA targeting KLK10 effectively suppressed the transcription of KLK10 in HT29 cells. Furthermore, si-KLK10 transfection also muted the protein expression of KLK10 (Figure 2B). Functional assay confirmed that KLK10 knockdown notably inhibited cell viability of colorectal cancer cells relative to the control group (Figure 2C). Simul-
A

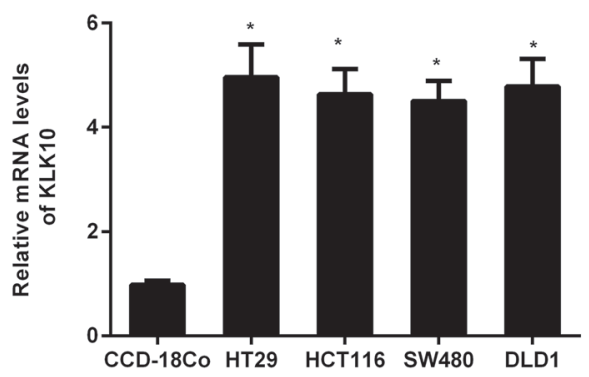

B

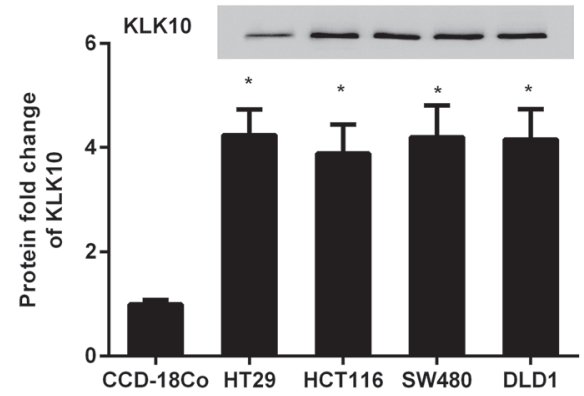

Figure 1. Elevation of KLK10 expression in CRC cells. A) The mRNA levels of KLK10 were determined in normal human colorectal epithelial cell CCD$18 \mathrm{Co}$ and four CRC lines (HT29, SW480, DLD1, and HCT116) by qRT-PCR. B) Protein expression of KLK10 in these cells was evaluated by western blotting assay. ${ }^{*} \mathrm{p}<0.05$ vs. CCD-18Co group 

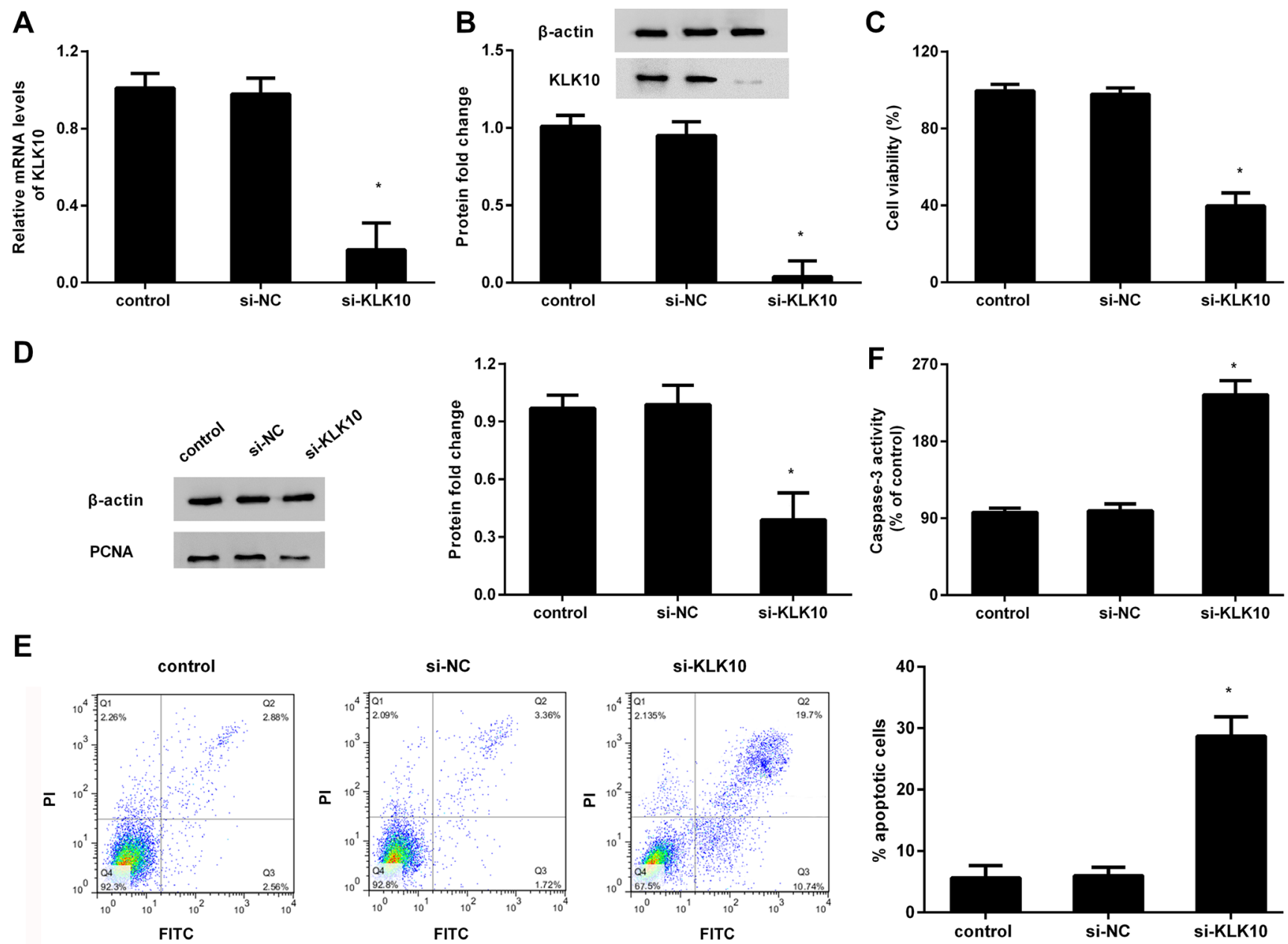

Figure 2. Knockdown of KLK10 suppressed cell viability and induced cell apoptosis in CRC cells. A) HT29 cells were transfected with si-KLK10. Approximately $48 \mathrm{~h}$ later, the efficacy of the KLK10 transcript was detected. B) The effect of si-KLK10 transfection on KLK10 protein expression. C) Cell viability was assessed by MTT assay when cells were treated with si-KLK10. D) Protein levels of proliferation-related PCNA were measured by western blotting. E) Cells were preconditioned with si-KLK10 and then stained with Annexin V-PI. Cell apoptosis was subsequently evaluated by flow cytometry. F) Caspase- 3 activity was determined using commercial kits. ${ }^{*} \mathrm{p}<0.05$ vs. control group

taneously, targeting KLK10 also restrained the protein expression of PCNA, a known marker for cell proliferation (Figure 2D). Further analysis found that, in contrast to the control group, KLK10 cessation increased cell apoptosis by $28.7 \%$ (Figure 2E). Concomitantly, caspase-3 activity was also elevated after KLK10 depression relative to the control group (Figure 2F).

Cessation of KLK10 affords the inhibitory effects on glycolytic metabolism in colorectal cancer cells. Glucose metabolism is widely accepted as a major contributor to cancer development. Next, we elucidated whether KLK10 influenced glucose metabolism in CRC. As presented in Figure $3 \mathrm{~A}$, the inhibition of KLK10 decreased glucose uptake in contrast to the control group. Moreover, cells transfected with si-KLK10 dramatically suppressed lactate production from $73.5 \mu \mathrm{M}$ to $30.2 \mu \mathrm{M}$ in HT29 cells (Figure $3 \mathrm{~B}$ ), indicating the inhibitor effects on lactate exportation.
Furthermore, KLK10 knockdown also reduced intracellular ATP contents (Figure 3C). Moreover, qRT-PCR and western blotting analysis confirmed that the cessation of KLK10 also depressed the mRNA (Figure 3D) and protein (Figure 3E) expression of glycolysis-related Glut1. These data indicate that KLK10 may facilitate glycolysis of CRC cells.

KLK10 depression regulates the PI3K/AKT/mTOR pathway. To elucidate the mechanism lying beneath KLK10-mediated function in CRC cell growth and glycolysis, the pathway of PI3K/AKT-mTOR was explored. The western blotting analysis found that the knockdown of KLK10 dramatically depressed the expression of phosphorylated AKT (p-AKT) relative to control group (Figures $4 \mathrm{~A}$ and 4B). Moreover, KLK10 suppression also inhibited the expression of p-mTOR (Figures $4 \mathrm{~A}$ and $4 \mathrm{C}$ ). No obvious changes were observed in the protein expression of AKT 

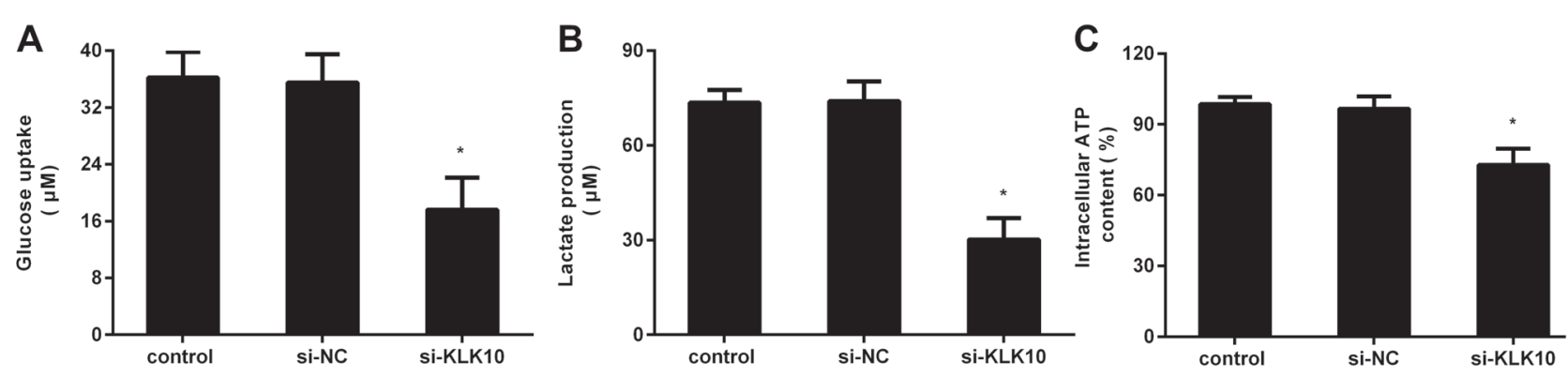

D

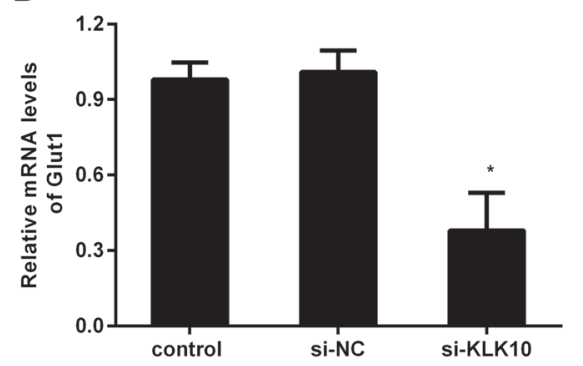

E

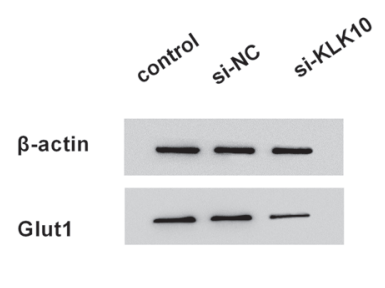

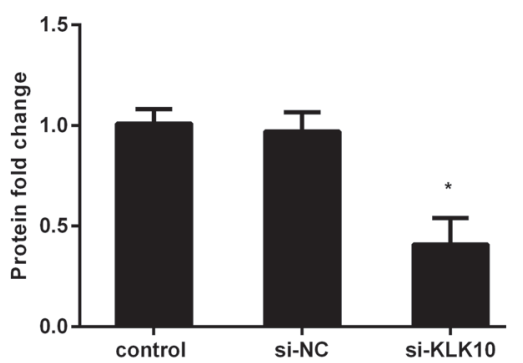

Figure 3. KLK10 cessation suppressed glucose metabolism in HT29 cells. A) The specific siRNA targeting KLK10 was transfected into HT29 cells; glucose uptake was then performed using a commercial assay kit. B) The effect of KLK10 depression on lactate production was evaluated. C) The contents of intracellular ATP were measured. D) Following transfection with si-KLK10, qRT-PCR was conducted to detect the mRNA levels of Glut1. E) The protein expression of Glut 1 was measured by western blotting. ${ }^{*} \mathrm{p}<0.05$

A

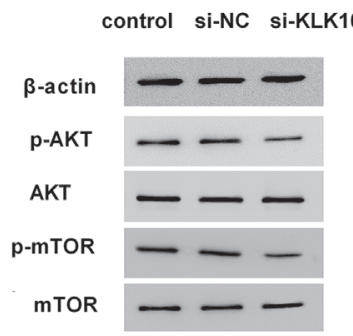

C

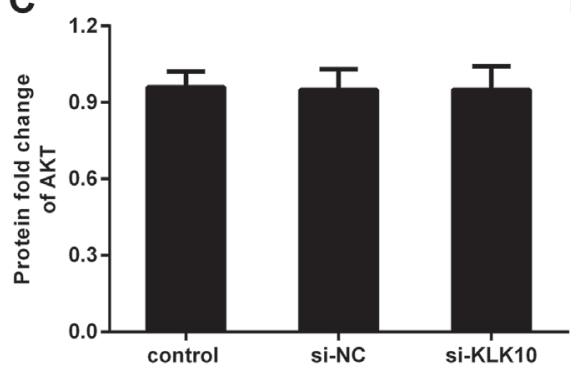

B

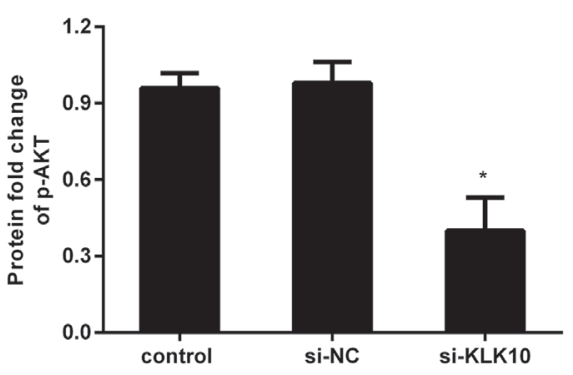

D

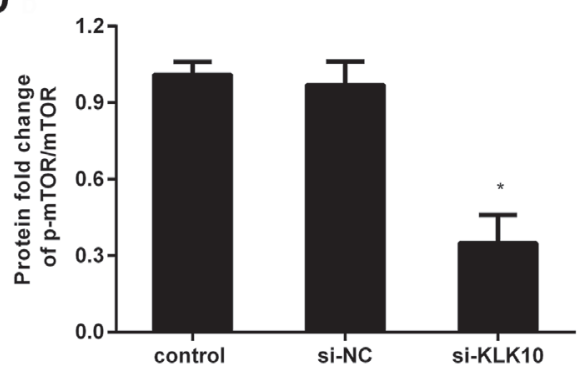

Figure 4. The knockdown of KLK10 restrained the activation of the PI3K/AKT/mTOR signaling. A) Cells were transfected with si-KLK10, and then the expressions of p-AKT, KAT, p-mTOR, and mTOR were determined by western blotting assay. B-D) The corresponding quantified analysis was conducted by ImageJ software. ${ }^{*} \mathrm{p}<0.05$

and mTOR (Figures $4 \mathrm{~A}$ and $4 \mathrm{D}$ ). Together, these results suggest that the deletion of KLK10 mutes the activation of PI3K/AKT-mTOR signaling.

Restoring the PI3K/AKT/mTOR signaling reverses the adverse role of KLK10 knockdown in CRC cell growth and glycolysis. We subsequently elucidated whether the PI3K/
AKT/mTOR pathway was implicated in KLK10-mediated regulation of cell growth and glucose metabolism in CRC cells. Preconditioning with PI3K/AKT activator IGF-1 notably overturned the adverse effects of KLK10 downregulation on p-AKT (Figures $5 \mathrm{~A}$ and $5 \mathrm{~B}$ ) and p-mTOR expression (Figures 5A and 5C). Further analysis substan- 
tiated that reactivating the PI3K/AKT-mTOR pathway via IGF-1 increased cell viability in cells with KLK10 deletion (Figure 5D). However, restoring this pathway decreased KLK10 inhibition-evoked apoptosis (Figure 5E) and caspase-3 activity (Figure 5F). Additionally, pretreatment with IGF-1 also reversed the suppressive roles of KLK10 cessation in glucose uptake (Figure 5G) and lactate production (Figure 5H).

Blocking the PI3K/AKT/mTOR pathway further aggravates the effects of KLK10 deletion on CRC cell growth and glycolysis. Next, we further muted the PI3K/AKT-mTOR pathway by preconditioning with the PI3K/AKT inhibitor LY294002 or mTOR antagonist rapamycin in KLK10knockdown cells (Figures 5A-5C). Noticeably, blocking the pathway of PI3K/AKT-mTOR by LY294002 or rapamycin both further aggravated the adverse effects of KLK10 cessation on cell viability (Figure 5D), but enhanced KLK10 inhibition-evoked cell apoptosis (Figure 5E) and caspase-3 activity (Figure 5F). Intriguingly, blocking the PI3K/AKT/ mTOR pathway further promoted KLK10 downregulationsuppressed glucose uptake (Figure 5G) and lactate production (Figure $5 \mathrm{H}$ ).
A

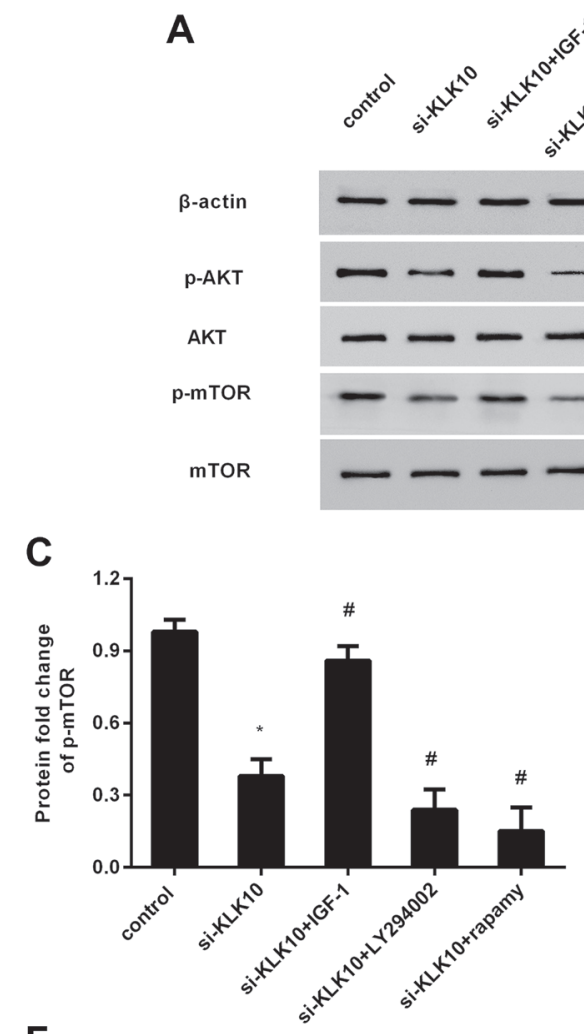

$\mathbf{F}$

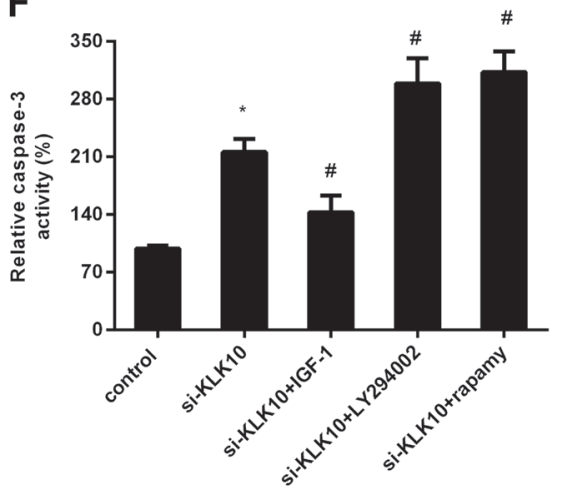

D

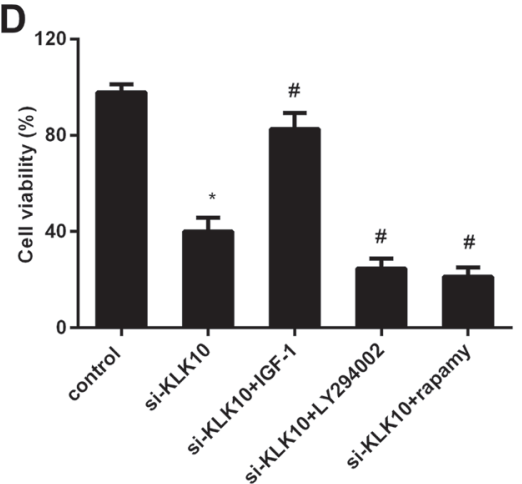

G

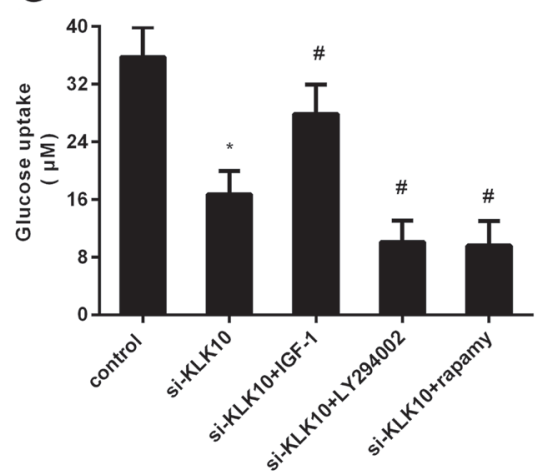

B

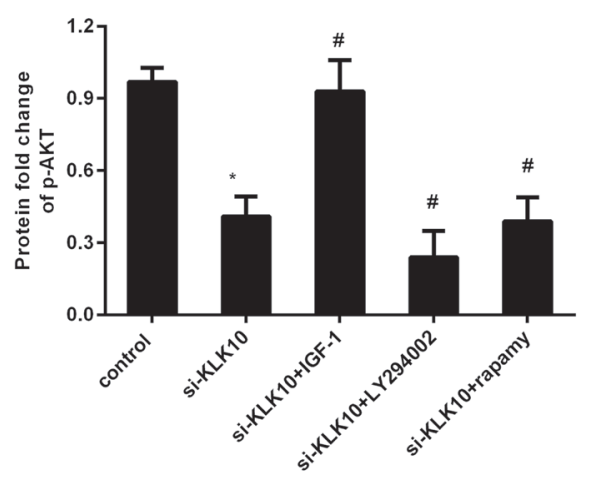

E

$\mathrm{H}$
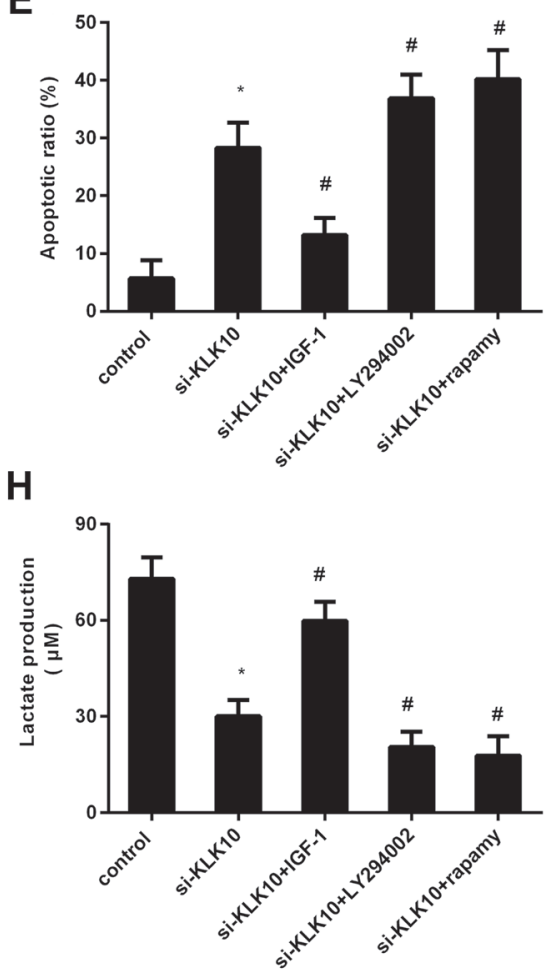

Figure 5. KLK10 regulated cell growth and glycolysis in CRC cells by the PI3K/AKT/mTOR signaling. A) HT29 cells transfected with si-KLK10 were pretreated with the PI3K/AKT activator IGF-1, PI3K/AKT inhibitor LY294002, or mTOR antagonist rapamycin. Then, the protein expression of pAKT and p-mTOR were determined. B, C) The quantified analysis of p-AKT (B) and p-mTOR (C) were performed by the ImageJ software. D-F) The effects on cell viability (D), apoptosis (E), and caspase-3 activity (F) were measured. G, H) The subsequent effects on glycolysis were also assessed by detecting glucose uptake $(\mathrm{G})$ and lactate release $(\mathrm{H}) .{ }^{*} \mathrm{p}<0.05$ vs. control group; ${ }^{*} \mathrm{p}<0.05$ vs. si-KLK10-treated group 


\section{Discussion}

Recent research confirms a negative correlation between high expression of KLK10 and overall survival rate in patients with CRC $[16,17]$. Nevertheless, its roles in the progression of $\mathrm{CRC}$ are poorly elucidated. The present findings corroborated the elevated levels of KLK10 in four CRC cell lines. Moreover, knockdown of KLK10 dramatically suppressed cell viability and evoked apoptosis. Simultaneously, KLK10 cessation also attenuated glucose metabolism. Noticeably, blocking the PI3K/AKT/mTOR signaling accounted for KLK10 depression-inhibited CRC cell growth and glucose metabolism. Therefore, KLK10 may act as an oncogene to facilitate the development of CRC by elevating cell growth and glycolytic metabolism.

Abundant evidence confirms abnormal expression of KLK10 in several carcinomas, such as breast cancer, esophageal cancer, and ovarian cancer $[14,18]$. KLK10 has been identified as a diagnostic and prognostic marker in ovarian cancer, colorectal cancer, breast cancer, and gastric cancer $[17,19,20]$. As a common KLK family member, KLK10 can act as a multifunctional regulator for various biological processes of cancer cells, including cell apoptosis, invasion, and chemotherapy resistance. Nevertheless, its function in carcinogenesis is controversial in several carcinomas [13, 14]. Intriguingly, the present study confirmed that targeting KLK10 restrained CRC cell proliferation, but increased cell apoptosis and caspase-3 activity. These findings suggest that KLK10 may act as an oncogene to facilitate CRC cell growth. Similarly, aberrant elevation of KLK10 promotes cell invasion and metastasis in pancreatic ductal adenocarcinoma [13]. Analogously, KLK10 elevation enhances trastuzumab resistance in gastric cancer [12]. However, KLK10 is recognized as a putative tumor suppressor in prostate cancer as the fact that KLK10 overexpression decelerates tumor proliferation and induces cell apoptosis [15]. Intriguingly, knockdown of KLK10 also incurs in cell cycle arrest and increases cisplatininduced apoptosis in esophageal cancer cells [14].

Metabolic reprogramming is widely defined as a proverbial feature of carcinoma, concomitant with the increased rate of glycolysis. Glucose is a major energy source for the generation of RNA and DNA. To maintain high rates of proliferation, cancer cells will undergo aberrant glucose metabolism that is essential for cell growth and invasion $[6,21]$. During this process, high levels of glycolysis will result in enhanced glucose consumption and lactate production, contributing to the development of malignancies. Recently, increasing evidence has supported a promising approach for cancer therapy by abrogating the abnormal glucose metabolism signaling. For instance, suppressing the glucose metabolic pathway by targeting protein kinase CK2 blunts bladder cancer cell survival [22]. In gastric cancer, blocking glucose metabolism can restrain cell growth and thus may offer a new strategy for cancer therapy [23]. In this study, cessation of KLK10 inhibited glycolytic metabolism including glucose uptake and lactate production. In tumor cells, glycolysis leads to ATP rapid accumulation that is essential for the energy requirement of cancer cell proliferation and tumor growth [24]. Noticeably, KLK10 knockdown also suppressed intracellular ATP contents in CRC cells. Glut1 is a well-known glycolysis-related element that can metabolize glucose to produce ATP. A recent study corroborates that Glut1 often acts as a glucose transporter to regulate cancer cell proliferation and invasion-metastasis potential by mediating glycolysis in various carcinomas $[8,21,25]$. Intriguingly, the current data substantiated that KLK10 depression decreased the expression of Glut1. Hence, these findings indicate that KLK10 inhibition may blunt cancer cell growth by suppressing glucose metabolism in CRC.

We next elucidated the molecular mechanism lying beneath KLK10-mediated CRC cell growth and glycolysis, and corroborated that suppression of KLK10 muted the activation of the canonical signal pathway PI3K/AKT/mTOR. Accumulating evidence has supported the critical roles of PI3K/AKT/mTOR signaling in the various physiological progression of cancer, such as cell growth, invasion, and chemoresistance [26-28]. It has been documented that activating the PI3K/AKT/mTOR pathway facilitates cisplatin resistance in non-small cell lung cancer [26]. In breast cancer, PI3K/AKT/mTOR activation also promotes the reprogramming of glucose metabolism [27]. These findings prompted us to investigate whether the $\mathrm{PI} 3 \mathrm{~K} / \mathrm{AKT} / \mathrm{mTOR}$ was implicated in KLK10-regulated CRC cell growth and glycolysis. In accordance with our hypothesis, preconditioning with IGF-1, an agonist of the PI3K/ AKT pathway, notably reversed the adverse effects of KLK10 cessation on cell growth and glucose metabolism in CRC cells. Importantly, blocking the $\mathrm{PI} 3 \mathrm{~K} / \mathrm{AKT} / \mathrm{mTOR}$ pathway with the inhibitor of LY294002 or rapamycin both aggravated KLK10 suppression-inhibited cell growth and glycolysis.

In conclusion, the present study substantiated the high expression of KLK10 in CRC cells. More important, knockdown of KLK10 suppressed cancer cell growth and glucose metabolism by blocking the $\mathrm{PI} 3 \mathrm{~K} / \mathrm{AKT} / \mathrm{mTOR}$ signaling. Accordingly, these findings highlight a new insight into how KLK10 facilitates the development of CRC, supporting a potential avenue for the treatment of CRC. The hypoxic microenvironment is a proverbial feature of locally advanced solid tumors including CRC and constitutes a critical contributor to cancer development and survival outcomes [29, 30]. Thus, we will explore the function of KLK10 in CRC cells under hypoxia conditions in our next plan. Moreover, whether KLK10 overexpression exerts the reverse effects on CRC cell growth will also be investigated in our future.

\section{References}

[1] FAVORITI P, CARBONE G, GRECO M, PIROZZI F, PIROZZI RE et al. Worldwide burden of colorectal cancer: a review. Updates Surg 2016; 68: 7-11. https://doi.org/10.1007/ s13304-016-0359-y 
[2] CENTER MM, JEMAL A, SMITH RA, WARD E. Worldwide variations in colorectal cancer. CA Cancer J Clin 2009; 59: 366-378. https://doi.org/10.3322/caac.20038

[3] ARNOLD M, SIERRA MS, LAVERSANNE M, SOERJOMATARAM I, JEMAL A et al. Global patterns and trends in colorectal cancer incidence and mortality. Gut 2017; 66: 683-691. https://doi.org/10.1136/gutjnl-2015-310912

[4] GARRIDO-CASTRO AC, SAURI-NADAL T, MACARULLA-MERCAD T. New Targets and New Drug Development in Colorectal Cancer. Current Colorectal Cancer Reports 2014; 10: 288-295. https://doi.org/10.1007/s11888-0140235-4

[5] CAIRNS RA, HARRIS IS, MAK TW. Regulation of cancer cell metabolism. Nat Rev Cancer 2011; 11: 85-95. https://doi. org/10.1038/nrc2981

[6] XU H, ZENG Y, LIU L, GAO Q, JIN S et al. PRL-3 improves colorectal cancer cell proliferation and invasion through IL-8 mediated glycolysis metabolism. Int J Oncol 2017; 51: 12711279. https://doi.org/10.3892/ijo.2017.4090

[7] CHEN GQ, TANG CF, SHI XK, LIN CY, FATIMA S et al. Halofuginone inhibits colorectal cancer growth through suppression of Akt/mTORC1 signaling and glucose metabolism. Oncotarget 2015; 6: 24148-24162. https://doi.org/10.18632/ oncotarget. 4376

[8] SUN HW, YU XJ, WU WC, CHEN J, SHI M et al. GLUT1 and ASCT2 as Predictors for Prognosis of Hepatocellular Carcinoma. PLoS One 2016; 11: e0168907. https://doi. org/10.1371/journal.pone.0168907

[9] LOU Y, WANG C, TANG Q, ZHENG W, FENG Z et al. Paeonol Inhibits IL-1beta-Induced Inflammation via PI3K/Akt/ NF-kappaB Pathways: In Vivo and Vitro Studies. Inflammation 2017; 40: 1698-1706. https://doi.org/10.1007/s10753017-0611-8

[10] ZHENG Z, XU L, ZHANG S, LI W, TOU F et al. Peiminine inhibits colorectal cancer cell proliferation by inducing apoptosis and autophagy and modulating key metabolic pathways. Oncotarget 2017; 8: 47619-47631. https://doi. org/10.18632/oncotarget.17411

[11] ZHANG HF, WANG YC, HAN YD. MicroRNA34a inhibits liver cancer cell growth by reprogramming glucose metabolism. Mol Med Rep 2018; 17: 4483-4489. https://doi. org/10.3892/mmr.2018.8399

[12] TANG L, LONG Z, ZHAO N, FENG G, GUO X et al. NES1/ KLK10 promotes trastuzumab resistance via activation of PI3K/AKT signaling pathway in gastric cancer. J Cell Biochem 2018; 119: 6398-6407. https://doi.org/10.1002/ jcb. 26562

[13] CAO XY, ZHANG XX, YANG MW, HU LP, JIANG SH et al. Aberrant upregulation of KLK10 promotes metastasis via enhancement of EMT and FAK/SRC/ERK axis in PDAC. Biochem Biophys Res Commun 2018; 499: 584-593. https:// doi.org/10.1016/j.bbrc.2018.03.194

[14] CHO H, WALKER A, WILLIAMS J, HASTY KA. Study of osteoarthritis treatment with anti-inflammatory drugs: cyclooxygenase-2 inhibitor and steroids. Biomed Res Int 2015; 2015: 595273. https://doi.org/10.1155/2015/595273
[15] HU J, LEI H, FEI X, LIANG S, XU H et al. NES1/KLK10 gene represses proliferation, enhances apoptosis and downregulates glucose metabolism of PC3 prostate cancer cells. Sci Rep 2015; 5: 17426. https://doi.org/10.1038/srep17426

[16] TALIERI M, ALEXOPOULOU DK, SCORILAS A, KYPRAIOS D, ARNOGIANNAKI N et al. Expression analysis and clinical evaluation of kallikrein-related peptidase 10 (KLK10) in colorectal cancer. Tumour Biol 2011; 32: 737744. https://doi.org/10.1007/s13277-011-0175-4

[17] ALEXOPOULOU DK, PAPADOPOULOS IN, SCORILAS A. Clinical significance of kallikrein-related peptidase (KLK10) mRNA expression in colorectal cancer. Clin Biochem 2013; 46: 1453-1461. https://doi.org/10.1016/j.clinbiochem.2013.03.002

[18] YANG X, ASAKAWA T, HAN S, LIU L, LI W et al. Neuroserpin Protects Rat Neurons and Microglia-Mediated Inflammatory Response Against Oxygen-Glucose Deprivation- and Reoxygenation Treatments in an In Vitro Study. Cell Physiol Biochem 2016; 38: 1472-1482. https://doi. org/10.1159/000443089

[19] PETRAKI C, YOUSSEF YM, DUBINSKI W, LICHNER Z, SCORILAS A et al. Evaluation and prognostic significance of human tissue kallikrein-related peptidase 10 (KLK10) in colorectal cancer. Tumour Biol 2012; 33: 1209-1214. https:// doi.org/10.1007/s13277-012-0368-5

[20] JIAO X, LU HJ, ZHAI MM, TAN ZJ, ZHI HN et al. Overexpression of kallikrein gene 10 is a biomarker for predicting poor prognosis in gastric cancer. World J Gastroenterol 2013; 19: 9425-9431. https://doi.org/10.3748/wjg.v19.i48.9425

[21] WANG Y, ZHANG X, WANG Z, HU Q, WU J, et al. LncRNA-p23154 promotes the invasion-metastasis potential of oral squamous cell carcinoma by regulating Glut1-mediated glycolysis. Cancer Lett 2018; 434: 172-183. https://doi. org/10.1016/j.canlet.2018.07.016

[22] ZHANG X, YANG X, YANG C, LI P, YUAN W et al. Targeting protein kinase CK2 suppresses bladder cancer cell survival via the glucose metabolic pathway. Oncotarget 2016; 7: 87361-87372. https://doi.org/10.18632/oncotarget.13571

[23] LIN LL, HSIA CR, HSU CL, HUANG HC, JUAN HF. Integrating transcriptomics and proteomics to show that tanshinone IIA suppresses cell growth by blocking glucose metabolism in gastric cancer cells. BMC Genomics 2015; 16: 41. https://doi.org/10.1186/s12864-015-1230-0

[24] ALLISON KE, COOMBER BL, BRIDLE BW. Metabolic reprogramming in the tumour microenvironment: a hallmark shared by cancer cells and $\mathrm{T}$ lymphocytes. Immunology 2017; 152: 175-184. https://doi.org/10.1111/imm.12777

[25] XIAO H, WANG J, YAN W, CUI Y, CHEN Z et al. GLUT1 regulates cell glycolysis and proliferation in prostate cancer. Prostate 2018; 78: 86-94. https://doi.org/10.1002/pros.23448

[26] GONG T, CUI L, WANG H, WANG H, HAN N. Knockdown of KLF5 suppresses hypoxia-induced resistance to cisplatin in NSCLC cells by regulating HIF-1alpha-dependent glycolysis through inactivation of the PI3K/Akt/mTOR pathway. J Transl Med 2018; 16: 164. https://doi.org/10.1186/s12967018-1543-2 
[27] YANG L, HOU Y, YUAN J, TANG S, ZHANG H et al. Twist promotes reprogramming of glucose metabolism in breast cancer cells through PI3K/AKT and p53 signaling pathways. Oncotarget 2015; 6: 25755-25769. https://doi.org/10.18632/ oncotarget.4697

[28] KAMAL A, LAKSHMA NAYAK V, NAGESH N, VISHNUVARDHAN MV, SUBBA REDDY NV. Benzo[b]furan derivatives induces apoptosis by targeting the PI3K/Akt/ mTOR signaling pathway in human breast cancer cells. Bioorg Chem 2016; 66: 124-131. https://doi.org/10.1016/j. bioorg.2016.04.004
[29] VADDE R, VEMULA S, JINKA R, MERCHANT N, BRAMHACHARI PV et al. Role of hypoxia-inducible factors (HIF) in the maintenance of stemness and malignancy of colorectal cancer. Crit Rev Oncol Hematol 2017; 113: 22-27. https:// doi.org/10.1016/j.critrevonc.2017.02.025

[30] LEE JH, JUNG S, PARK WS, CHOE EK, KIM E et al. Prognostic nomogram of hypoxia-related genes predicting overall survival of colorectal cancer-Analysis of TCGA database. Sci Rep 2019; 9: 1803. https://doi.org/10.1038/s41598-01838116-y 